\title{
¿Y SI PRESCRIBIMOS ARTE? EL PAPEL DEL ARTE EN LA MEJORA DE LA SALUD Y EL BIENESTAR
}

\section{What if we prescribe art? The role of the arts in improving health and well-being}

\author{
Elena GUARDIOLA; Josep-E BAÑOS \\ e-mail: josepeladi.banos@uvic.cat \\ Facultad de Medicina. Universitat de Vic - Universitat Central de Catalunya.
}

Fecha de recepción: 24 de mayo de 2020

Fecha de aceptación: 27 de mayo de 2020

Fecha de publicación: 15 de septiembre de 2020

Es obvio: el médico debe ser buen médico desde el punto de vista técnico. Pero también lo es que esto solo no es suficiente. Y es aquí donde, sobre todo en las últimas décadas, se ha considerado en medicina el binomio arte-ciencia ${ }^{1}$. Así, en el siglo XXI se está produciendo un renacimiento de las humanidades médicas en el currículum médico, con nuevos programas y la definición de un amplio modelo para incluir historia, filosofía, literatura y arte, al tiempo que se considera que las humanidades son un recurso pedagógico útil e interesante para la formación de los estudiantes de ciencias de la salud: literatura, teatro, poesía, narrativas o historias de vida, ópera, cine, pintura, etc. se presentan como herramientas que pueden ayudar al estudiante en su formación ${ }^{2}$. Esto ha dado lugar a publicaciones diversas, muchas de las cuales recientemente en esta misma revista, que han considerado el uso del $\operatorname{arte}^{3}$ y las humanidades $^{1,4}$, la literatura ${ }^{2,5}$ y el cine ${ }^{6}$, con distintos enfoques sobre este tema.

En paralelo, también en las últimas décadas, se ha estudiado el valor potencial del arte dentro del proceso terapéutico y curativo de la enfermedad. Hace unos meses reflexionábamos desde aquí sobre la influencia que la lectura puede tener sobre nuestra salud ${ }^{7}$ y otro artículo se adentraba en sus efectos terapéuticos ${ }^{8}$. Aunque se ha tratado la relación entre arte y salud desde hace décadas ${ }^{9}$, últimamente se han publicado muchos artículos 
sobre los efectos beneficiosos del arte y su impacto en la salud y en la calidad de vida ${ }^{10-12}$, tanto en adolescentes ${ }^{13}$ como en personas mayores que viven en residencias ${ }^{14}$ o que están enfermas ${ }^{15}$, en personas con demencia ${ }^{16} \mathrm{o}$ en pacientes con distintas enfermedades psiquiátricas ${ }^{17}$.

En las últimas dos décadas se ha incrementado el interés en investigar los efectos del arte en la salud y el bienestar. Al mismo tiempo, en diversos países europeos se han iniciado acciones encaminadas a favorecer esta relación. En este contexto, en noviembre de 2019, la Oficina Regional de la OMS para Europa publicó un primer informe en el que examinaba la evidencia existente sobre el efecto de las actividades artísticas en la salud ${ }^{18}$. En este informe se incluyen los resultados de una revisión de más de 3.000 estudios y se examina la evidencia de las actividades artísticas en la promoción de la salud.

El informe de la $\mathrm{OMS}^{18}$ proporciona una revisión exhaustiva de los beneficios para la salud de la participación, activa o pasiva, en cinco amplias categorías: artes escénicas (p. ej., actividades relacionadas con la música, danza, teatro, canto o cine); artes visuales, diseño y artesanía (p. ej., manualidades, diseño, pintura, fotografía o escultura), literatura (p. ej., escribir, leer o participar en encuentros literarios), cultura (p. ej., ir a museos, galerías, exposiciones de arte, conciertos, teatro o festivales culturales) y arte en línea, en formatos digitales y electrónicos (p. ej., animaciones, realización de películas y diseños por ordenador). Estas categorías combinan compromiso activo y receptivo y, lo que es también muy importante, trascienden las fronteras culturales y permiten flexibilidad para que se desarrollen nuevas formas de manifestaciones artísticas.

En general, los hallazgos del estudio muestran que el arte puede impactar potencialmente tanto en la salud física como mental de las personas. Los resultados de la revisión se agrupan en dos grandes temas: prevención y promoción, y gestión y tratamiento ${ }^{18}$. En relación con la prevención y la promoción, los resultados muestran cómo el arte puede afectar a los determinantes sociales de la salud, apoyar el desarrollo infantil, alentar comportamientos que promueven la salud, ayudar a prevenir la mala salud y apoyar el cuidado y el autocuidado. Dentro del manejo y el tratamiento de la enfermedad el arte puede ayudar a las personas que padecen enfermedades mentales, apoyar en la atención a personas con afecciones agudas, ayudar a las personas con trastornos neurológicos y en el tratamiento de enfermedades no transmisibles y contribuir al cuidado al final de la vida.

Parece probado, por tanto, que existen vínculos entre el arte y la salud. Así, las actividades artísticas pueden considerarse como intervenciones complejas o multimodales en las que se combinan múltiples componentes diferentes que promueven la salud. Las actividades artísticas pueden comportar un compromiso estético, con participación de la imaginación, activación sensorial, evocación de la emoción y estimulación cognitiva. Y dependiendo de su naturaleza, una actividad artística también puede implicar interacción social, actividad física, compromiso con temas de salud y establecimiento de una relación con entornos de atención médica o entornos saludables ${ }^{19}$.

Por otro lado, los distintos componentes de las actividades artísticas pueden desencadenar respuestas psicológicas, fisiológicas, sociales y de comportamiento que están vinculadas con los resultados de salud. En una actividad artística están presentes componentes estéticos y emocionales que pueden favorecer la expresión y la regulación de las emociones y también la reducción del estrés ${ }^{20-22}$. Las emociones están íntimamente ligadas a la forma en que manejamos nuestra salud mental y el estrés es un factor de riesgo reconocido para el inicio o progresión de distintas enfermedades, como las cardiovasculares. Por otro lado, cuando se participa en actividades artísticas se produce una estimulación cognitiva que favorece el aprendizaje y el desarrollo de habilidades, se asocia con un menor riesgo de 
desarrollar demencias y también se interrelaciona con enfermedades como la depresión.

En muchas actividades artísticas se produce interacción social. Esto contribuye a reducir la soledad y favorece la presencia de apoyo social. La soledad y la falta de relaciones interpersonales están vinculadas con respuestas fisiológicas adversas, deterioro cognitivo, funcional y motor, enfermedades mentales y mortalidad prematura ${ }^{18,23,24}$. Otro aspecto a considerar es la actividad física que se realiza en determinadas actividades relacionadas con el arte, que pueden favorecer la reducción de comportamientos sedentarios, que están asociados con afecciones como dolor crónico, depresión y demencia.

$\mathrm{Si}$, además, las actividades artísticas se asocian a aspectos relacionados con la salud (p. ej., si se realizan en entornos de atención médica, programas artísticos a medida diseñados con objetivos específicos de salud o bienestar, o impartidos por profesionales especializados), se pueden fomentar comportamientos que mejoren la salud. A través de actividades que promuevan, por ejemplo, dietas saludables, la abstención del tabaco, el consumo moderado de alcohol o la visita al médico para revisiones o estudios de cribado, se pueden mejorar las condiciones de salud previas y reducir el riesgo de mortalidad por determinadas enfermedades ${ }^{18}$.

La revisón de la $\mathrm{OMS}^{18}$ concluye que, aunque hay una gran variedad de enfoques y metodologías, el arte juega un importante papel en la promoción de la salud, ayuda a prevenir la aparición de enfermedades mentales y deterioro físico asociado a la edad, apoya el tratamiento de enfermedades mentales, enfermedades no transmisibles y trastornos neurológicos, y es útil en la asistencia tanto de enfermedades agudas como al final de la vida. Asimismo, señala que, aunque algunos países han avanzado en el desarrollo de políticas sanitarias en las que las actividades artísticas se consideran un importante apoyo para la salud y el bienestar, muchos aún no han abordado las oportunidades que existen en el uso de las artes en este campo.
Por este motivo, plantea una serie de consideraciones para los países miembros de la Región Europea de la OMS con el fin de que se apoye el desarrollo de políticas o estrategias a largo plazo encaminadas a lograr una mayor colaboración y sinergia entre los sectores de la salud y las artes, que permita aprovechar su potencial para mejorar la salud global. El desarrollo de programas que implementen estas políticas redundaría en un beneficio mutuo. Para que esto sea posible es preciso apoyar la inclusión de la educación en artes y humanidades dentro de la formación de los profesionales de la salud; esto contribuirá a mejorar sus habilidades clínicas, personales y de comunicación.

Un editorial de The Lancet $^{25}$, en relación con esta revisión, destacaba el papel de las artes en la promoción de la salud y en la prevención de enfermedades. Así, explicaba, las representaciones positivas de enfermedades mentales en películas, en series de televisión o en representaciones teatrales pueden reducir el estigma y las actitudes negativas frente a estos enfermos, al tiempo que aumentan la comprensión de la enfermedad. Por otro lado, la participación en actividades artísticas puede reducir el riesgo de desarrollar enfermedades mentales al tiempo que aumenta la autoestima y puede proteger contra el deterioro cognitivo. Se ha visto, por ejemplo, que la música puede reducir la frecuencia cardíaca y la presión arterial antes de una intervención quirúrgica y disminuir la ansiedad, el dolor y el uso de analgésicos después de la operación. Mientras el canto puede ayudar a pacientes con enfermedades respiratorias, la música y el baile pueden ayudar a la rehabilitación motora después de un accidente cerebrovascular, el baile puede mejorar los trastornos motores de las personas con enfermedad de Parkinson y la música puede ayudar a los pacientes con demencia a participar en actividades de la vida diaria. Además de los beneficios para la salud y la promoción del bienestar ligado al arte, el riesgo de resultados negativos es muy bajo. El editorial ${ }^{25}$ 


\section{¿Y SI PRESCRIBIMOS ARTE? EL PAPEL DEL ARTE EN LA MEJORA DE LA SALUD Y EL BIENESTAR ELENA GUARDIOLA; JOSEP-E BAÑOS}

señala que una mayor inversión en investigación que evalúe el efecto de las artes en la salud podría generar retornos prometedores.

Por todo lo expuesto anteriormente, puede ser interesante, cuando no importante, que el médico "prescriba» la realización de actividades artísticas, en especial si además incluyen una relación social, ya que parece claro que pueden brindar beneficios adicionales a los tratamientos médicos convencionales.

\section{Referencias}

1. Guardiola E, Baños JE. El papel de las humanidades médicas en la educación de los profesionales de la salud del siglo XXI. Rev Med Cine. 2017; 13(4): 155-7.

2. Baños JE, Guardiola E. Sobre lo que los estudiantes de medicina pueden aprender en la literatura. Rev Med Cine. 2016; 12(2): 75-7.

3. Hidalgo Balsera A. Potencial contribución del arte al desarrollo de habilidades de observación. Rev Med Cine. 2020; 16(1): 59-60.

4. Baños JE, Cambra-Badii I. Las humanidades en los estudios de Medicina en España. Rev Med Cine. 2020; 16(1): 49-50.

5. Fresnadillo Martínez MJ. La literatura en los estudios de la salud. Rev Med Cine. 2020; 16(1): 51-2.

6. Farré $M$, Papaseit E, Pérez-Mañá C, Baños JE. El cine comercial como recurso para la docencia de la Farmacología. Rev Med Cine. 2020; 16(1): 55-6.

7. Guardiola E, Baños JE. La lectura ¿influye en nuestra salud? Rev Med Cine. 2017; 13(1): 1-2.

8. Hidalgo A, Cantabrana B. Efectos terapéuticos de la lectura. Rev Med Cine. 2017; 13(2): 75-88.

9. Sheeran I. Arts for health. BMJ. 1988; 297(6664): 1667-9.

10. Cavanagh B, Haracz K, Lawry M, James C. It's like another world: the perceived beneficial effects of an artistically designed multisensory environment. Med Humanit. 2019; 45(1): 52-9.
11. Howarth L. Creative health: the arts for health and wellbeing. Perspect Public Health. 2018; 138(1): 26-7.

12. Clift S. Creative arts as a public health resource: moving from practice-based research to evidencebased practice. Perspect Public Health. 2012; 132(3): 120-7.

13. Daykin N, Orme J, Evans D, Salmon D, McEachran $\mathrm{M}$, Brain S. The impact of participation in performing arts on adolescent health and behaviour: a systematic review of the literature. J Health Psychol. 2008; 13(2): 251-64.

14. Curtis A, Gibson L, O'Brien M, Roe B. Systematic review of the impact of arts for health activities on health, wellbeing and quality of life of older people living in care homes. Dementia (London). 2018; 17(6): 645-69.

15. Ford K, Tesch L, Dawborn J, Courtney-Pratt H. Art, music, story: The evaluation of a person-centred arts in health programme in an acute care older persons' unit. Int J Older People Nurs. 2018; 13(2): e12186.

16. Morse N, Chatterjee $\mathrm{H}$. Museums, health and wellbeing research: co-developing a new observational method for people with dementia in hospital contexts. Perspect Public Health. 2018; 138(3): 152-9.

17. Leckey J. The therapeutic effectiveness of creative activities on mental well-being: a systematic review of the literature. J Psychiatr Ment Health Nurs. 2011; 18(6): 501-9.

18. Fancourt D, Finn S. What is the evidence on the role of the arts in improving health and well-being? A scoping review. Health Evidence Network (HEN) synthesis report 67. Copenhagen: WHO Regional Office for Europe; 2019.

19. Fancourt D. Arts in health: designing and researching interventions. Oxford: Oxford University Press; 2017.

20. Juslin PN. From everyday emotions to aesthetic emotions: towards a unified theory of musical emotions. Phys Life Rev. 2013; 10(3): 235-66.

21. Fancourt D, Garnett C, Spiro N, West R, Müllensiefen D. How do artistic creative activities regulate our 


\section{¿Y SI PRESCRIBIMOS ARTE? EL PAPEL DEL ARTE EN LA MEJORA DE LA SALUD Y EL BIENESTAR ELENA GUARDIOLA; JOSEP-E BAÑOS}

emotions? Validation of the Emotion Regulation Strategies for Artistic Creative Activities Scale (ERS-ACA). PLoS One. 2019; 14(2): e0211362.

22. Mennin D, Farach F. Emotion and evolving treatments for adult psychopathology. Clin Psychol Sci Pract. 2007; 14(4): 329-52.

23. Boss L, Kang D-H, Branson S. Loneliness and cognitive function in the older adult: a systematic review. Int Psychogeriatr. 2015; 27(4): 541-53.
24. Steptoe A, Shankar A, Demakakos P, Wardle J. Social isolation, loneliness, and all-cause mortality in older men and women. Proc Natl Acad Sci U S A. 2013; 110(15): 5797-801.

25. The Lancet. Promoting and prescribing the arts for health. Editorial. Lancet. 2019; 394: 1880.

\begin{tabular}{|l|l|}
\hline & $\begin{array}{l}\text { Elena Guardiola es doctora en Medicina. Investigadora asociada de la } \\
\text { Facultad de Ciencias de la Salud y de la Vida de la Universitat Pompeu Fabra } \\
\text { (2007-2018) y desde } 2019 \text { de la Facultad de Medicina de la Universitat de } \\
\text { Vic-Universitat Central de Catalunya, se ha especializado en información, } \\
\text { documentación y redacción científica, áreas en las que ha impartido nume- } \\
\text { rosos cursos. Su interés por la relación entre la medicina y la literatura se ha } \\
\text { plasmado en la participación en varios proyectos así como en la publicación } \\
\text { de diversos trabajos. }\end{array}$ \\
\hline $\begin{array}{l}\text { Josep-Eladi Baños es doctor en Medicina y catedrático de universidad. Fue } \\
\text { profesor de Farmacología en la Universitat Autònoma de Barcelona (1985- } \\
\text { 2002) y en la Universitat Pompeu Fabra (2002-2019) donde fue vicerrector } \\
\text { de Docencia y Ordenación Académica (2005-2013). Ha recibido diversas } \\
\text { distinciones a la calidad de la innovación docente de la Generalitat de Cata- } \\
\text { lunya. Fue director del Grupo de Investigación Educativa en Ciencias de la } \\
\text { Salud (GRECS) de la Universitat Pompeu Fabra (2016-2018). En la actualidad } \\
\text { es catedrático de Farmacología clínica en la Universitat de Vic-Universitat } \\
\text { Central de Catalunya, donde es Rector desde 2019. }\end{array}$ \\
\hline
\end{tabular}

